\title{
ALGORITHM FOR CONVERGENCE CRITERIA SIMULATION ON LMS ADAPTIVE FILTERS
}

\author{
Andrés Frías-Velázquez, René de Jesús Romero-Troncoso \\ FIMEE, Universidad de Guanajuato, Tampico 912, Col. Bellavista, 36730 Salamanca Gto., México, \\ Email: kyrie_v@hotmail.com, troncoso@salamanca.ugto.mx
}

Abstract. A standard algorithm for LMS-filter simulation, tested with several convergence criteria under system identification configuration is presented in this paper. Establishing a reliable convergence criterion is mandatory in order to properly design an LMS filter and so avoid instability problems that may arise if both quantization effects and interdependency relationship among learning curve behaviors are not taken into account. Simulations performed under Matlab show remarkable differences between convergence criteria proposed by several authors and one presented in a previous work derived from a complete learning curve analysis.

\section{INTRODUCTION}

There is no doubt that the popularity of LMS (Least Mean Square) adaptive filters is due to their simplicity, easy hardware implementation and overall performance in most applications. Some applications of LMS adaptive filters include: system identification, $60 \mathrm{~Hz}$ noise suppression, channel equalization, and echo cancellation. Since LMS filtering was invented, several attempts have been made to establish more restrictive convergence criteria; however, most of these are derived just from one behavior of the learning curve: transient or steady-state behavior, instead from a formal stability analysis, which may lead us to undesirable results on the learning curve. Some of the most commonly used convergence criteria are: Widrow et al [1] consider that a necessary condition for convergence is that the weight error vector remains stable; on the other hand Haykin [2] formulates a criterion based on the transient behavior stability of the mean square error. Diniz [3] includes in his analysis the finite word length effects, establishing a lower limit to avoid the stopping effect and derives an upper limit based on the weight-error correlation matrix. Feuer and Weinstein [4] propose a more restrictive convergence criterion that guarantees the updating of the weight-error correlation matrix. In [5] a complete stability study is performed, derived from the learning curve behavior, finite word length effects, and a sensitivity analysis of the steady-state error excess.

Most of the tested convergence criteria in this paper are obtained using the system identification configuration and under similar analysis assumptions, therefore the obtained results may validate one of the proposed criteria in the present work.

\section{PROPOSED ALGORITHM}

In order to compare the performance of the learning curve using the different proposed convergence criteria, it is necessary to establish an algorithm that considers main practical aspects on the LMS filter implementation. Fig. 1 shows the detailed block diagram model for system identification, including the finite word length effects as additive noise. From Fig. $1 x(k)$ represents the input signal, which is assumed as wide band noise, zero mean, with mutually independent values, and generated by a wide sense stationary process. The vector $\mathbf{w}_{\mathrm{o}}=\left[w_{o 1} w_{o 2} \ldots w_{o N-1}\right]^{T}$ represents the impulse response of the unknown system. $\mathbf{w}(k)=\left[w_{0}(k) w_{1}(k) \ldots w_{N-1}(k)\right]^{T}$ represents the length $N$ weight vector at iteration $k$ obtained by the recursive equation described in (1). 


$$
\mathbf{w}(k+1)=\mathbf{w}(k)+2 \mu_{q} e(k) \mathbf{x}(k)+\boldsymbol{\eta}_{\mathbf{u}}(k)
$$

where $\mu_{q}$ is the quantized convergence factor, $e(k)$ is the error signal, $\mathbf{x}(k)=[x(k) x(k-1) \ldots x(k-N+1)]^{T}$ is the shifted input vector, and $\boldsymbol{\eta}_{\mathbf{u}}(k)$ is the quantization noise due to the product $2 \mu_{q} e(k) \mathbf{x}(k)$. Uniform distribution perturbations $\eta_{d}(k)$ and $\eta_{y}(k)$ are due to the quantization performed after the sum of the inner products between the input vector and the corresponding weight vector. Signal $n(k)$ is commonly known as measurement noise.

The different tested convergence criteria are shown in table 1. Each criterion is derived according to many stability considerations, as a result, it is necessary to calculate several statistical parameters such as $\sigma_{x}^{2}$, which represents the input signal variance, $\sigma_{e}^{2}$ is the quantization noise variance of the error signal, $\sigma_{n}^{2}$ is the measurement noise variance, $n_{v}$ is the linear limit with $v$ degrees of freedom defined in [5], $b_{c}$ and $B$ are the number of bits of the quantizers after the sign bit, and $\gamma_{2}$ is the kurtosis excess.

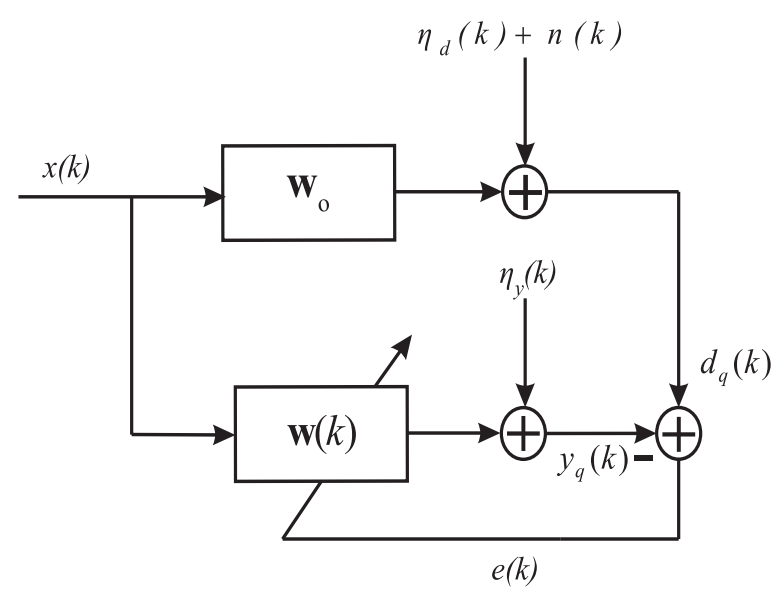

Fig. 1 Quantized LMS model for system identification problem.

\begin{tabular}{lc}
\hline \multicolumn{1}{c}{ Reference } & Range \\
\hline Widrow et al. [1] & $0<\mu<\frac{1}{\lambda_{\max }}$ \\
Haykin [2] & $<\mu<\frac{2}{\operatorname{tr}[\mathbf{R}]}$ \\
Diniz [3] & $\frac{2^{-b_{c}}}{4 \sigma_{x} \sqrt{\sigma_{e}^{2}+\sigma_{n}^{2}}}<\mu<\frac{1}{\operatorname{tr}[\mathbf{R}]}$ \\
Feuer and Weinstein [4] & $0<\mu<\frac{1}{3 \operatorname{tr}[\mathbf{R}]}$ \\
Frías and Romero[5] & $\frac{2^{-B}}{2 \sigma_{x} \sqrt{\sigma_{n}^{2}+\sigma_{e}^{2}}}<\mu_{q} \leq \frac{1}{n_{v}\left(\operatorname{tr}[\mathbf{R}]+\left(\gamma_{2}+2\right) \lambda\right)}$ \\
\hline
\end{tabular}

Note: Where $\operatorname{tr}[\mathbf{R}]=N \sigma_{x}^{2}$ and $\lambda=\sigma_{x}^{2}$.

Table 1 Operating ranges proposed by several authors.

\section{RESULTS}

The LMS filter model shown in Fig. 1 is simulated using white noise input $\left(\gamma_{2}=0\right)$ with variance $\sigma_{x}^{2}=1.5625$, measurement noise variance $\sigma_{o}^{2}=0.01$, filter order $N=10$, ensemble of 300 independent runs, and rounding quantizers of $b_{c}=7$ bits or equivalently $B=8$ bits. The algorithm is evaluated to verify its performance under extreme conditions like low bit resolution due to, in this case, the relationship between lower and upper limit of the convergence factor is more evident, and as a result we get a more restrictive operating range. The following results evaluate the upper limits of the convergence criteria using values of $\mu$ close to those limits, under the next precedence order

$$
\frac{2^{-b_{e}}}{4 \sigma_{x} \sqrt{\sigma_{e}^{2}+\sigma_{n}^{2}}}<\mu_{1}<\frac{1}{3 \operatorname{tr}[\mathbf{R}]}<\mu_{2}<\frac{1}{2\left(\operatorname{tr}[\mathbf{R}]+\left(\gamma_{2}+2\right) \lambda\right)}<\mu_{3}<\frac{1}{\operatorname{tr}[\mathbf{R}]}<\mu_{4}<\frac{2}{\operatorname{tr}[\mathbf{R}]}<\mu_{5}<\frac{1}{\lambda_{\max }}
$$

It is important to mention that the upper limit proposed by [5] is a movable limit, which is set for this simulation to the maximum allowable value: 10 degrees of freedom.

Fig. 2a shows simulations of the learning curve with values of $\mu$ close to the upper limits proposed by Widrow [1] and Haykin [2], we can conclude that using these limits the learning curve becomes instable 
and obviously it is not reached the optimal error. Fig. 2b shows simulations of the learning curve with more restrictive criteria, which let us evaluate the advantages and disadvantages of each criterion, for instance, using the criterion proposed by Frías [5], the learning curve converges faster than the established by Feuer [4], on the contrary the steady-state error in [5] is slightly greater than [4]; in spite of the differences, both approaches remain stable. When Diniz criterion is used, the learning curve converges slowly and steady-state error increases, also an instability issue is present, which is caused by high sensitivity of steady-state error excess to input signal statistical changes, as demonstrated in [5].

As a consequence of the simulations, we can prove that convergence rate has a quadratic behavior and Frías criterion works close to the maximum rate, since for greater and smaller values to the stated by Frías [5] the convergence rate is smaller. Even though the maximum linear limit proposed in [5] may fail under specific conditions, we can establish an upper limit more restrictive with less degrees of freedom.

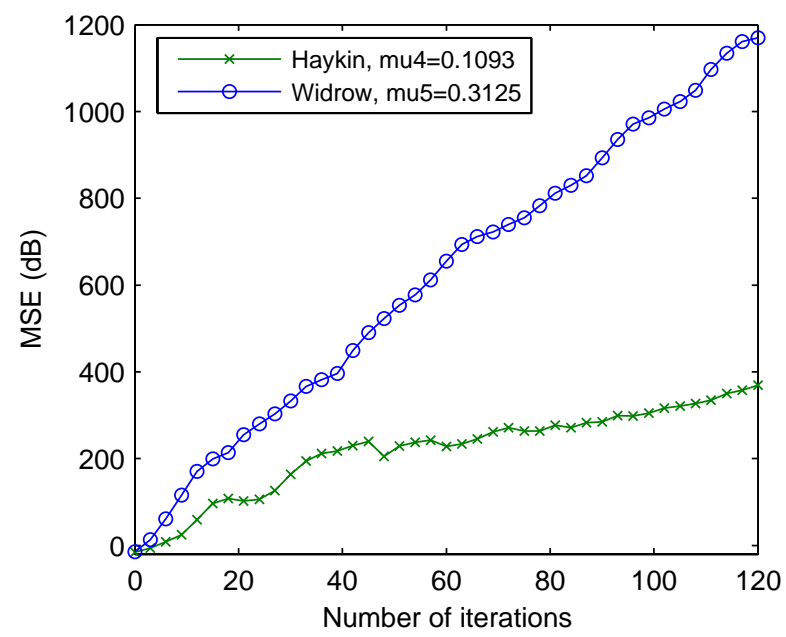

Fig. 2a Learning curves using Widrow and Haykin criteria

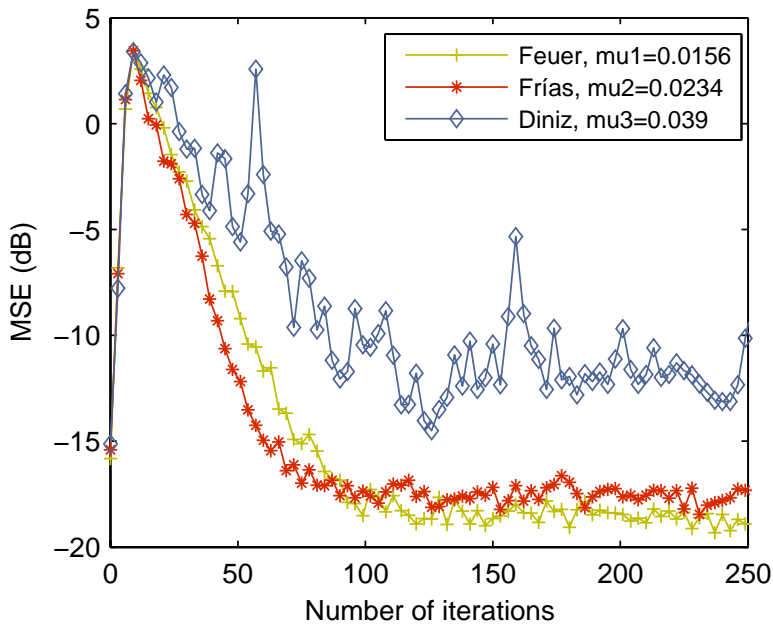

Fig. 2b Learning curves using Diniz, Frías and Feuer criteria.

\section{CONCLUSIONS}

A practical algorithm for the analysis of the LMS-filter learning curve using several convergence criteria was proposed in this paper. The performance of an LMS-filter is clearly affected by several issues as described through this work; therefore, it is necessary to count on reliable design tools that guarantee an optimal hardware implementation, in this sense, we can conclude that the criterion stated in [5] assures the stability of the LMS algorithm, takes into account the finite word length effects, and it is based on both behaviors of the learning curve.

\section{REFERENCES}

[1] B. Widrow et al., "Stationary and nonstationary learning characteristics of the LMS adaptive filter", Proc. IEEE, 64 (8), 1151-1162, 1976.

[2] S. Haykin, Adaptive Filters Theory, Prentice Hall, New Jersey, 1996.

[3] P.S.R. Diniz, Adaptive Filtering: Algorithms and Practical Implementation, Kluwer Academic Publishers, Dordrecht, The Netherlands, 2002.

[4] A. Feuer, E. Weinstein, "Convergence analysis of LMS filters with uncorrelated gaussian data", IEEE Trans. Acoust. Speech Signal Process., 33 (1), 222-230, 1985.

[5] A. Frías-Velázquez, R.J. Romero-Troncoso, "Finite word length effect analysis on the adaptive filter learning curve with LMS algorithm", Signal Processing, under review. 